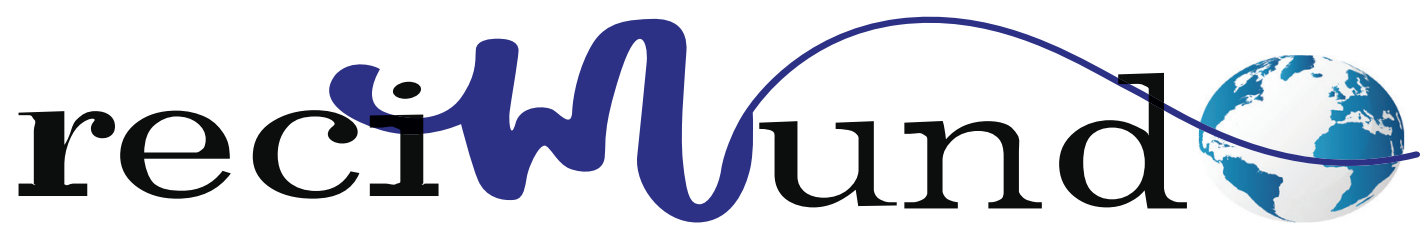

Revista Científica Mundo de la Investigación y el Conocimiento

DOI: 10.26820/recimundo/5.(4).oct.2021.181-190

URL: https://recimundo.com/index.php/es/article/view/1318

EDITORIAL: Saberes del Conocimiento

REVISTA: RECIMUNDO

ISSN: 2588-073X

TIPO DE INVESTIGACIÓN: Artículo de revisión

CÓDIGO UNESCO: 3213.13 Ortodoncia-Estomatología

PAGINAS: $181-190$

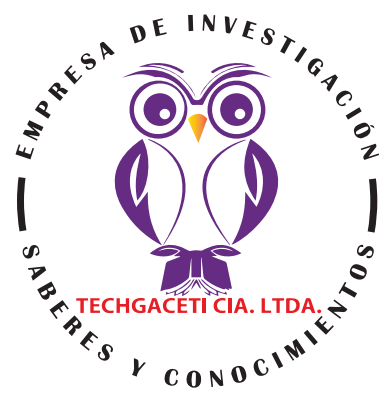

\title{
Disfunción craneomandibular y su relación con la ortodoncia u oclusión
}

Craniomandibular dysfunction and its relationship with orthodontics or occlusion

Disfunção craniomandibular e sua relação com a ortodontia ou oclusão

Julio Ildefonso Rosero Mendoza'; Fanny Alicia Mendoza Rodríguez; Carly Lorena Núñez García3;

Jacobo César Rosero Mendoza ${ }^{4}$

RECIBIDO: 02/09/2021 ACEPTADO: 20/09/2021 PUBLICADO: 30/10/2021

1. Magister en Gerencia Hospitalaria; Especialista en Ortodoncia; Odontólogo; Universidad de Guayaquil, Facultad Piloto; Guayaquil, Ecuador; julio.roserom@ug.edu.ec; (iD https://orcid.org/0000-0001-5326-3320

2. Magister en Diseño Curricular; Diplomado en Docencia Superior; Doctora en Odontología; Universidad de Guayaquil, Facultad Piloto; Guayaquil, Ecuador; fanny.mendozar@ug.edu.ec; (iD https://orcid.org/0000-0002-5791-5872

3. Odontóloga de la Universidad de Guayaquil, Investigadora Independiente; Guayaquil, Ecuador; carlylorena@outlook.com; (iD) https://orcid.org/0000-0003-3908-9295

4. Especialista Rehabilitación Oral; Odontólogo; Guayaquil, Ecuador; jacoborosero@hotmail.com; (iD https://orcid.org/00000002-4162-8385

CORRESPONDENCIA

Julio Ildefonso Rosero Mendoza

julio.roserom@ug.edu.ec

Guayaquil, Ecuador

(C) RECIMUNDO; Editorial Saberes del Conocimiento, 2021 


\section{RESUMEN}

Las alteraciones que se originan en el sistema masticatorio, se conocen como disfunciones craneomandibuares. Este tipo de alteraciones, producen diferentes molestias en el oído, cuello, la mandíbula o la espalda afectando la articulación temporomandibular y los músculos que se encargan de la movilidad de dicha articulación. Incide en los músculos masticatorios y los músculos de la cabeza y cuello siendo estos los encargados de mantener la cabeza en una posición adecuada. Los tratamientos para la corrección de los defectos e irregularidades en la mordida como la ortodoncia, en ocasiones, tienen una relación con la presencia de este tipo de trastornos. La elección de la posición de referencia mandibular en el tratamiento de la ortodoncia esta intrínsecamente relacionada con la aparición de disfunciones craneomandibulares. En tal sentido, el desarrollo del trabajo literario se enfoca en la prevalencia de los trastornos craneomandibulares, su etiología y fisiopatología, oclusión, tratamiento de ortodoncia y DCM. Adicionalmente, la posición de referencia mandibular en la ortodoncia, tomando en consideración que el ortodoncista es quien tiene la responsabilidad de adoptar una referencia mandibular adecuada a sus pacientes respetando el equilibrio que existe en el sistema estomatognático.

Palabras clave: Disfunción craneomandibular, trastornos, ortodoncia, articulación temporomandibular.

\section{ABSTRACT}

The alterations that originate in the masticatory system are known as craniomandibular dysfunctions. These types of alterations produce different discomfort in the ear, neck, jaw or back, affecting the temporomandibular joint and the muscles that are responsible for the mobility of said joint. It affects the chewing muscles and the muscles of the head and neck, which are responsible for keeping the head in a proper position. Treatments for the correction of defects and irregularities in the bite, such as orthodontics, are sometimes related to the presence of these types of disorders. The choice of the mandibular reference position in orthodontic treatment is intrinsically related to the appearance of craniomandibular dysfunctions. In this sense, the development of the literary work focuses on the prevalence of craniomandibular disorders, their etiology and pathophysiology, occlusion, orthodontic treatment and DCM. Additionally, the mandibular reference position in orthodontics, taking into consideration that the orthodontist is the one who has the responsibility of adopting an adequate mandibular reference for his patients, respecting the balance that exists in the stomatognathic system.

Keywords: Craniomandibular dysfunction, disorders, orthodontics, temporomandibular joint.

\section{RESUMO}

As alterações originadas no sistema mastigatório são conhecidas como disfunções craniomandibulares. Esses tipos de alterações produzem diferentes desconfortos na orelha, pescoço, mandíbula ou nas costas, afetando a articulação temporomandibular e os músculos responsáveis pela mobilidade dessa articulação. Afeta os músculos da mastigação e os músculos da cabeça e do pescoço, responsáveis por manter a cabeça na posição correta. Tratamentos para correção de defeitos e irregularidades na mordida, como a ortodôntica, às vezes estão relacionados à presença desses tipos de distúrbios. A escolha da posição de referência mandibular no tratamento ortodôntico está intrinsecamente relacionada ao aparecimento de disfunções craniomandibulares. Nesse sentido, o desenvolvimento da obra literária enfoca a prevalência das doenças craniomandibulares, sua etiologia e fisiopatologia, oclusão, tratamento ortodôntico e CMD. Além disso, a posição de referência mandibular em Ortodontia, levando-se em consideração que o ortodontista é quem tem a responsabilidade de adotar uma referência mandibular adequada para seus pacientes, respeitando o equilíbrio que existe no sistema estomatognático.

Palavras-chave: Disfunção craniomandibular, distúrbios, ortodontia, articulação temporomandibular. 


\section{Introducción}

Las disfunciones craneomandibulares (DCM) y su relevancia para la ortodoncia han sido un tema muy debatido en los últimos años. Este tipo de trastornos se relacionan con el malestar de la articulación temporomandibular (ATM).

El trastorno es multifactorial con un grado de influencia psicógena que varía a lo largo de la vida de un individuo con fases de síntomas que afectan la calidad de vida. En un intento por tratar este complejo grupo de trastornos, se han propuesto varios relatos psicofisiológicos y psicológicos, pero ninguno de ellos pudo dilucidar claramente una correlación directa con la DCM (Michelotti \& Lodice, 2010).

La DCM ha sido difícil de definir. A lo largo de los años se han utilizado diferentes términos para referirse a la variedad de síntomas que se relacionan con la ATM y, en consecuencia, incluso en un solo individuo, el diagnóstico de disfunción de la ATM depende de la definición utilizada. Sin embargo, de acuerdo con Okeson \& Leeuw, (2011), "el término disfunción craneomandibulares (DCM) se refiere a un grupo de afecciones musculoesqueléticas que ocurren en la región temporomandibular, caracterizadas por dolor en los músculos de la masticación y en la ATM, o ambos". Estas perturbaciones contribuyen al deterioro de la calidad de vida de los pacientes, así como a su funcionamiento social.

Anteriormente, se creía que estos trastornos podían tratarse mediante principios oclusales gnatológicos. Sin embargo, "existen diferencias fundamentales entre Ios abordajes gnatológicos y neuromusculares en terapia a la hora de abordar las necesidades de los pacientes que presentan los numerosos signos y síntomas que comprometen al paciente con disfunción craneomandibular" (Michelotti \& Lodice, 2010). Por lo tanto, "surgió un nuevo enfo- que, denominado modelo biopsicosocial; es más científico y ampliamente aceptado por la comunidad científica dental ya que sus explicaciones se basan en un enfoque médico-cognitivo" (Okeson \& Leeuw, 2011).

El objetivo de este estudio es contribuir a una mejor comprensión de la naturaleza de los trastornos craneomandibulares mediante la revisión bibliográfica, en aras de busca enfocar la literatura sobre la elección de la posición de referencia mandibular (posición intercuspídea, posición de relación céntrica o posición terapéutica) en base a consideraciones clínicas y teniendo en cuenta el equilibrio del sistema estomatognático.

\section{Metodología}

Para el desarrollo de este proceso investigativo, se plantea como metodología la encaminada hacia una orientación científica particular que se encuentra determinada por la necesidad de indagar en forma precisa y coherente una situación, en tal sentido Davila, (2015) define la metodología "como aquellos pasos anteriores que son seleccionados por el investigador para lograr resultados favorables que le ayuden a plantear nuevas ideas" (p.66)

Lo citado por el autor, lleva a entender que el desarrollo de la acción investigativa busca simplemente coordinar acciones enmarcadas en una revisión bibliográfica con el fin de complementar ideas previas relacionadas Disfunción craneomandibular y su relación con la ortodoncia u oclusión a través de una revisión de literatura, para así finalmente elaborar un cuerpo de consideraciones generales que ayuden a ampliar el interés propuesto.

\section{Tipo de Investigación}

Dentro de toda práctica investigativa, se precisan acciones de carácter metodológi-

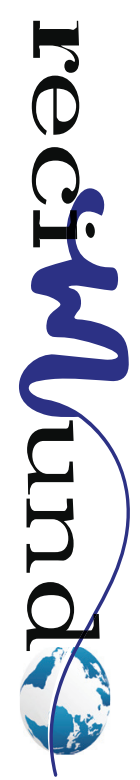


co mediante las cuales se logra conocer y proyectar los eventos posibles que la determinan. En este sentido, la presente investigación corresponde al tipo documental, definido por Castro (2016), "se ocupa del estudio de problemas planteados a nivel teórico, la información requerida para abordarlos se encuentra básicamente en materiales impresos, audiovisuales y / o electrónicos". (p.41).

En consideración a esta definición, la orientación metodológica incluye la oportunidad de cumplir con una serie de actividades inherentes a la revisión y lectura de diversos documentos, donde se encuentran ideas explicitas relacionadas con los tópicos encargados de identificar una característica inmersa en el estudio. Por lo tanto, se realizaron continuas interpretaciones con el claro propósito de revisar aquellas apreciaciones propuestas por diferentes investigadores en relación al tema de interés, para luego dar la respectiva argumentación a los planteamientos, en función a las necesidades encontradas en la investigación, apoyados en las herramientas tecnológicas para la búsqueda de trabajos con valor científico disponibles en la web que tenían conexión con el objetivo principal de la investigación.

\section{Fuentes Documentales}

El análisis correspondiente a las características que predomina en el tema seleccionado, llevan a incluir diferentes fuentes documentales encargadas de darle el respectivo valor científico y en ese sentido cumplir con la valoración de los hechos a fin de generar nuevos criterios que sirven de referencia a otros procesos investigativos. Para Castro,(2016) las fuentes documentales incorporadas en la investigación documental o bibliográfica, "representa la suma de materiales sistemáticos que son revisados en forma rigurosa y profunda para llegar a un análisis del fenómeno" (p.41). Por lo tanto, se procedió a cumplir con la lectura previa determinada para encontrar aquellos aspectos estrechamente vinculados con el tema, con el fin de explicar mediante un desarrollo las respectivas apreciaciones generales de importancia.

\section{Técnicas para la Recolección de la Infor- mación}

La conducción de la investigación para ser realizada en función a las particularidades que determinan a los estudios documentales, tiene como fin el desarrollo de un conjunto de acciones encargadas de llevar a la selección de técnicas estrechamente vinculadas con las características del estudio. Bolívar, (2015), refiere, que es "una técnica particular para aportar ayuda a los procedimientos de selección de las ideas primarias y secundarias". (p.71).

Tal como lo expresa, Bolívar, (2015) "Las técnicas documentales proporcionan las herramientas esenciales y determinantes para responder a los objetivos formulados y llegar a resultados efectivos" (p. 58). Es decir, para responder con eficiencia a las necesidades investigativas, se introdujeron como técnica de recolección el método inductivo, que hizo posible llevar a cabo una valoración de los hechos de forma particular para llegar a la explicación desde una visión general. El autor Bolívar, (2015) tambien expresa que las técnicas de procesamiento de datos en los estudios documentales "son las encargadas de ofrecer al investigador la visión o pasos que deben cumplir durante su ejercicio, cada una de ellas debe estar en correspondencia con el nivel a emplear" (p. 123). Esto indica, que para llevar a cabo el procesamiento de los datos obtenidos una vez aplicadas las técnicas seleccionadas, tales como: fichas de resumen, textual, registros descriptivos entre otros, los mismos se deben ajustar al nivel que ha sido seleccionado. 


\section{Resultados}

Las disfunciones craneomandibulares representan un trastorno significativo a nivel mundial, "el $75 \%$ de la población tiene al menos un signo de DMC (movimiento anormal de la mandíbula, ruidos articulares, dolor a la palpación, etc.) y aproximadamente el 33\% tiene al menos un síntoma (dolor facial, articulaciones, entre otros.)" (Bourzgui, 2012). Los autores también expresan que, "entre el 85 y el 95\% de la población presentará uno o más síntomas de DCM en su vida, y entre el 5 y el $6 \%$ de la población notificará dolor de mandíbula relacionado con DCM clínicamente significativo".

Las DCM afectan a niños, adolescentes y adultos. Los autores Campos, Herrera, \& Ruan, (2006) encontraron que las DCM están presentes en "16-25\% de los niños, $30 \%$ de los adolescentes y $60 \%$ de los adultos, sin embargo, este tipo de anomalías se presenta en niños de diferente edades, encontrándose aumento en la prevalencia de acuerdo al aumento en la edad del niño (Campos, Herrera, \& Ruan, 2006.).

\section{Etiología y fisiopatología de la DCM}

La etiología y fisiopatología de este tipo de anomalías son poco conocidas, esto se debe a que la fluctuación de los síntomas con sucesivos periodos de activación y remisión que dificultan su estudio, por lo cual los diferentes factores en la DCM aún no están claros. A lo largo de los años, se han ofrecido muchos esquemas de clasificación para los factores de DCM. Los autores Boever, Carlsson, \& Klineberg, (2000) expresan, entre las clasificaciones que se utilizan con frecuencia se distingue de la siguiente manera:

- factores predisponentes que aumentan el riesgo de DCM: factores estructurales (patrones oclusales, pérdida de calibración, etc.), calidad tisular, enfermedades sistémicas, edad, tipología facial y bruxismo;

- factores desencadenantes: macrotrauma o microtraumatismo, bruxismo y exceso de capacidad de tolerancia articular;

- factores de perpetuación: en su mayoría desatendidos pero generalmente dominados por el estado conductual, social y emocional, tienden a ser más predominantes.

El sistema estomatognático es una estructura complicada y los pacientes suelen adaptarse a su dimensión vertical de oclusión existente. "Cuando se excede la capacidad de compensación, las estructuras débiles como los dientes, los músculos y las articulaciones ceden y la enfermedad se manifiesta" (Bourzgui, 2012). De la misma forma, Winocur \& Gavish, (2001) concluyen que "las hiperfunciones relacionadas con hábitos parafuncionales como el bruxismo o el uso de chicle contribuyen significativamente a la aparición de dolor y ruido en las articulaciones".

La oclusión juega un papel causal significativo en la DCM. La creencia en esta relación causal se basó originalmente en la observación clínica directa. No obstante, Badel \& Marotti, (2012) dicen que "no encontraron ningún apoyo sólido para una etiología oclusal de la DMC, al menos no como un factor único o dominante", por lo que "estimaron que la contribución de la oclusión a la DMC es mínima y en la mayoría de los casos no supera el 10-20\%" (Badel \& Marotti, 2012.).

Adicionalmente, otros estudios revelaron que no existe una relación causal entre la oclusión y la DCM. Además, señalaron que debido a fallas en el diseño de la investigación, la asociación causal entre la oclusión dental y la ATM no se ha invalidado y sigue siendo una pregunta abierta donde gran parte, si no la mayor parte, de la confusión sobre el papel de la oclusión está profundamente arraigada en la falta de aprecia-

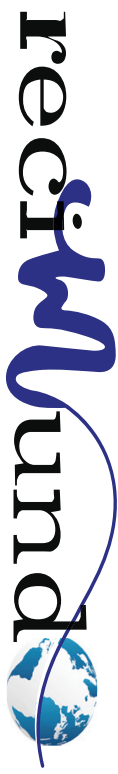


ción de los problemas en la inferencia causal (Kirveskari \& Alanen, 2009).

Hoy en día, existe una buena evidencia científica de que el papel de la oclusión no debe sobrevalorarse para evitar el sobrediagnóstico y el sobretratamiento. Por tanto, "debe seguir siendo un componente importante de las prácticas terapéuticas y puede constituir uno de los principales factores de desarrollo del sistema estomatognático" (Turp \& Schindler, 2012).

La posible asociación entre el tratamiento ortodóncico, ortopédico u ortoquirúrgico y la DMC ha sido con frecuencia un tema de debate entre los médicos en las últimas décadas. A pesar de la gran cantidad de estudios, aún son inciertas muchas dudas sobre la participación real del tratamiento de ortodoncia en la etiología, prevención y tratamiento de la DMC.

Por lo tanto, la mayoría de los investigadores coinciden en la ausencia de una relación causal entre la ortodoncia y la DMC. De hecho, una serie de condiciones (es decir, descoordinación muscular, relación inestable disco-cóndilo y alteraciones óseas) pueden interferir con la relación oclusal y con el análisis de ortodoncia (Bourzgui, 2012).

Según Conti, Oltramari, \& Navarro, (2007), la DCM puede desarrollarse "durante el tratamiento de ortodoncia; sin embargo, no hay evidencia de que la mecánica de ortodoncia pueda exponer al sujeto a un mayor riesgo de DMC, y hay poca evidencia de que el tratamiento de ortodoncia pueda prevenir la DCM". Además, Ios autores mostraron que el tratamiento de ortodoncia realizado durante la adolescencia "no puede aumentar ni disminuir el riesgo de desarrollar DCM más adelante. Esto es válido independientemente de la mecánica que se utilice: con o sin extracciones y con o sin aparatos ortopédicos" (Conti, Oltramari, \& Navarro, 2007).

\section{Signos y síntomas de disfunción cra- neomandibular (DCM)}

De acuerdo con SEDCYDO, (2015) los síntomas que se asocian a este tipo de afección se relacionan con "alteraciones dentarias, palpaciones dolorosas, signos de disfunción biomecánica con dificultad en la apertura y cierre de la boca, entre otros". A continuación se detalla cada uno:

Alteraciones dentarias:

- Desgastes de las superficies oclusales o cortantes de los dientes.

- Indentaciones en los bordes laterales de la lengua.

- Línea blanca en la mucosa yugal (cara interna de la mejilla).

Palpación dolorosa:

- En la musculatura de la cara, cuello y espalda.

- En la articulación temporomandibular (ATM), ya sea en reposo o en movimiento.

Signos de disfunción biomecánica, con dificultad en la apertura y cierre de la boca:

- Limitación de la apertura.

- Desviación al abrir hacia un lado u otro.

- Ruidos o crujidos articulares a la palpación de la ATM al abrir o cerrar la boca.

Adicionalmente, signos y síntomas de ansiedad, depresión, sobrecarga por estrés, cuando van unidos a los síntomas dentarios o articulares descritos anteriormente. Signos de hiperelasticidad de los tejidos periarticulares de varias articulaciones del cuerpo (SEDCYDO, 2015).

Dolores de cabeza de tipo opresivo en la zona frontal, temporal o parietal de la cabeza (cefaleas tensionales relacionadas con el apretamiento de los dientes). Dolores de cuello, espalda y hombros, asocia- 
do a alteraciones de la postural corporal. Dolor de oídos, por otalgia refleja debida a una compresión de la ATM a consecuencia de una sobrecarga funcional mantenida por apretamiento dentario. Pueden coexistir síntomas de sensación vertiginosa, hipoacusia y tinnitus, sobre todo cuando la exploración otorrinolaringológica previa ha sido negativa. Hormigueo o dolor mandibular matutino debido la presencia de apretamiento y/o rechinamiento nocturno de los dientes bruxismo (SEDCYDO, 2015).

\section{El tratamiento de ortodoncia y su rela- ción con DCM}

Los ortodoncistas deberían poder manejar tales situaciones clínicas, basando su trabajo en evidencia científica y considerando el aspecto multifactorial de tal problema. También deben poder distinguir pacientes con riesgo y pacientes sin riesgo. "Durante la intervención, deben optar por criterios que favorezcan la estabilidad oclusal manteniendo sus funciones. Además, el tratamiento de ortodoncia se considera una terapia oclusal que debe realizarse con la posición de referencia mandibular para la reconstrucción de la oclusión" (Decker \& Kohault, 2008).

El sistema de referencia evalúa los cambios realizados en relación con el estado inicial, pero también para transferir información del clínico al laboratorio y viceversa. Pero la pregunta que se plantea es la siguiente: ¿qué referencia elegir durante el tratamiento de ortodoncia especialmente en presencia de DCM? Este tema ha suscitado una considerable controversia.

El concepto de referencia implica una situación reproducible y registrable, que no se ve afectada por el tratamiento propuesto. Los autores Orthlieb, Brocard, \& Schittly, (2006) "ofrecen tres posibilidades en este contexto, la posición oclusal intercuspal, posición oclusal de relación céntrica y la posición terapéutica mandibular".

\section{Posición oclusal intercuspiana (POI)}

Esta es la posición mandibular que implica el contacto entre los dientes al tragar. En esta posición, hay un número infinito de posiciones condilares en la cavidad glenoidea.

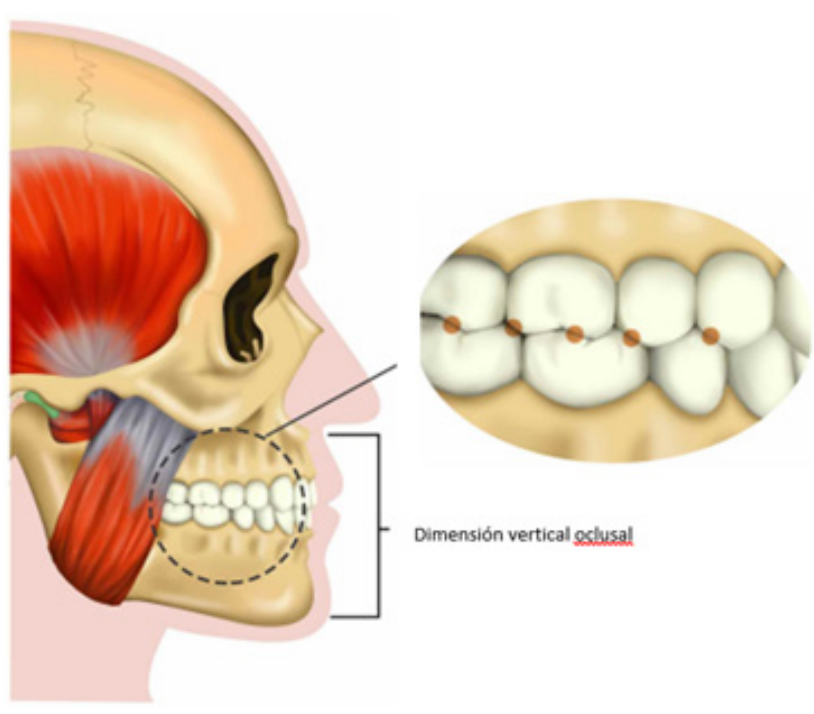

Figura 1. Posición de Máxima Intercuspidación (PMI), definida por el determinante anatómico dentario. Esta posición determina la Dimensión Vertical Oclusal.

Nota. (Venegas, Farfán, \& Fuentes, 2021)

\section{Relación céntrica ( $R C)$}

La relación céntrica se define como "la relación de la mandíbula con el maxilar cuando los cóndilos se encuentran en sus posiciones más posteriores no tensas en la fosa glenoidea" (López, Gómez, \& Canseco, 2004). Sin embargo, según Orozco \& Arroyo, (2008), "la definición de relación céntrica ha cambiado durante el último medio siglo de una posición de cóndilo retruido, posterior y, en su mayor parte, superior a una posición de cóndilo anterosuperior". La RC se utiliza para restaurar pacientes desdentados con prótesis removibles o implantosoportadas bien sean híbridas o fijas. Debido a que el dentista quiere poder relacionar de manera reproducible el

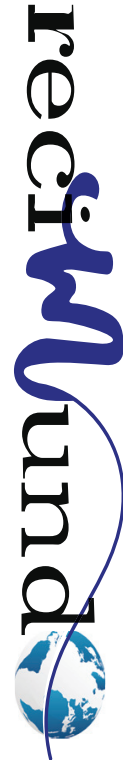


maxilar y la mandíbula del paciente, pero el paciente no tiene dientes con los que establecer su propia dimensión vertical de oclusión, se ha ideado otro método para lograr este objetivo. El cóndilo solo puede estar en el mismo lugar en el que estaba la última vez que lo colocó el dentista (Orozco \& Arroyo, 2008).

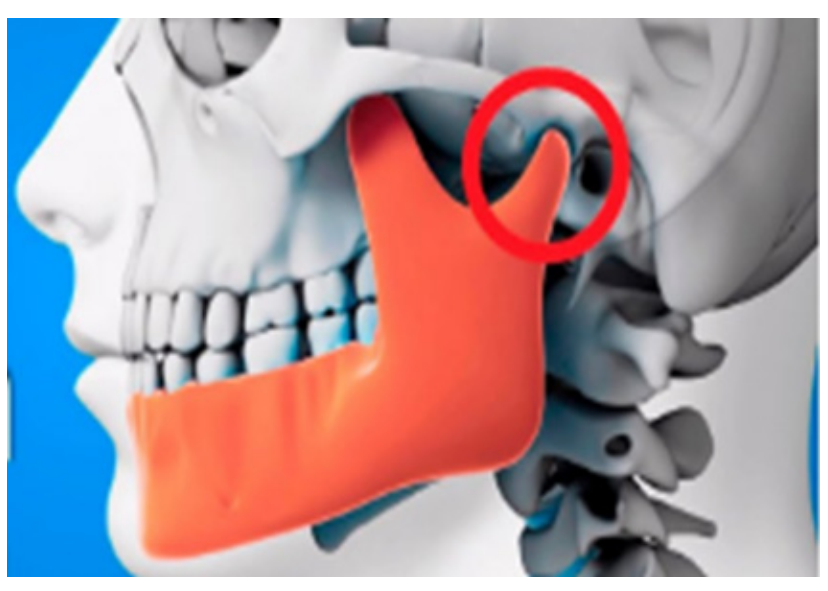

Figura 2. Relación Céntrica.

Nota. (Gutiérrez, 2020)

Los creyentes de la relación céntrica, "afirman la relación de la mandíbula con el maxilar cuando los conjuntos cóndilo-disco correctamente alineados están en la posición más superior frente a las eminentes, independientemente de la dimensión vertical oclusal (DVO) o la posición del diente" (López, Gómez, \& Canseco, 2004). Los conceptos de relación céntrica han sido reemplazados en gran medida por conceptos de odontología neuromuscular que se consideran mucho más fisiológicos.

En la posición más superior, los conjuntos cóndilo-disco se refuerzan medialmente, por lo que la relación céntrica también es la posición más media.

Un conjunto cóndilo-disco correctamente alineado en relación céntrica puede resistir la carga máxima de los músculos elevadores sin signos de incomodidad. La definición de relación céntrica puede cambiar con una mayor comprensión del movimiento mandibular. Cada individuo tiene una posición apropiada para él, y no existe una posición única de relación céntrica normal (Orozco \& Arroyo, 2008).

Tratamiento elegir: Cambio de posición céntrica (PC) o posición oclusal intercuspiana (POI).

El abordaje con ortodoncia se considera que está asociado con una rehabilitación oclusal completa. . Por lo tanto, "el diagnóstico y el tratamiento solo pueden realizarse mediante CR para lograr la coordinación entre la oclusión y la función masticatoria que muestre si el paciente es sintomático o no" (Orozco \& Arroyo, 2008).

Según Conti, Oltramari, \& Navarro, (2007), "la relación céntrica $(\mathrm{RC})$ es la posición de los maxilares en la que los cóndilos tienen una posición ortopédica estable". Por lo tanto, para cualquier cambio de posición céntrica (PC), la posición oclusal intercuspal (POI) que provoque cambios en la relación sagital intercalada, el diagnóstico y el tratamiento deben basarse en el análisis en RC. "La POI sólo se utilizará si dicta la posición mandibular mediante un máximo de contactos estabilizadores y extendidos armoniosamente en una posición cercana a la relación céntrica sin diferencial transversal" (Orozco \& Arroyo, 2008).

Sin embargo, en pacientes con DMC, el uso de la PC es cuestionable, ya que se ha definido para un sistema estomatognático asintomático. No obstante, Rinchuse \& Kandasamy, (2006) distinguen dos enfoques en el tratamiento de ortodoncia, gnatológico y no gnatológico, y concluyen que la posición condilar en la fosa no condiciona la aparición del DCM y el montaje del articulador, así como la determinación de armonizar la RC y la POI provoca muy poco o ningún beneficio en ortodoncia ( $p$. 498). 
La posición neuromuscular se encuentra entre la POI y la RC en la dirección anteroposterior. Por lo tanto, "la posición de la POI puede tomarse como punto de partida para la posición de equilibrio neuromuscular, porque es más fácil de realizar y reduce los costos de procesamiento y el tiempo empleado en el tratamiento de ortodoncia (Hamata, Zuim, \& Garcia, 2009).

\section{Conclusión}

A pesar de las investigaciones durante décadas y el estado de los avances de la medicina actual, la disfunción cranemandibular no parece estar relacionada con el tratamiento de ortodoncia y la corrección en la mordida u oclusión, así como tampoco se puede considerar la aparición o prevención de este tipo de afección. Hoy en día aún existe mucha controversia literatura sobre la referencia más confiable en ortodoncia y su relación con la existencia de DCM o en el abordaje de la ortodoncia como tratamiento.

En tal sentido, es necesario estar atento al examinar y tratar a cada paciente antes, durante y después del tratamiento de ortodoncia, especialmente cuando los factores de riesgo dominan el cuadro clínico. Por eso, cuando el ortodoncista se enfrenta a la presencia de signos, síntomas o problemas relacionados con alteraciones articulatorias internas, debe tratar estas alteraciones antes de continuar el tratamiento, especialmente pueden ser la causa de trastornos morfológicos en pacientes jóvenes.

En este caso, los medios reversibles no invasivos siguen siendo los métodos más apropiados para su uso. Durante el tratamiento, el ortodoncista debe adoptar una referencia mandibular acorde a cada paciente y respetar el equilibrio existente en el sistema estomatognático, con la finalidad de evitar en un futuro molestias en las articulaciones que se relacionan con la corrección de la mordida, tomando en consideración que cada paciente es un caso particular.

\section{Bibliografía}

Badel, J., \& Marotti, M. (2012.). Temporomandibular disorders and occlusion. Acta Clinica Croatica, 51(3), 419-424.

Boever, J., Carlsson, G., \& Klineberg, I. (2000). Need for occlusal therapy and prosthodontic treatment in the management of temporomandibular disorders. Part I. Occlusal interferences and occlusal adjustment. Journal of Oral Rehabilitation, 27(5), 367-379. Recuperado el 21 de Oct de 2021

Bolívar, J. (2015). Investigación Documental. México. Pax.

Bourzgui, F. (2012). Basic Aspects and Clinical Considerations, (Part 4. Temporomandibular Disorder and Orthodontic:). InTech, Rijeka, 327-376. Recuperado el 21 de Oct de 2021

Campos, M., Herrera, A., \& Ruan, V. (2006.). Desordenes Témporomandibulares en la población infantil. Un tema controversial - Revisión Bibliográfica. Revista Latinoamericana de Ortodoncia y Odontopediatria. Recuperado el 21 de Oct de 2021, de Obtenible en: www.ortodoncia.ws

Castro, J. (2016). Técnicas Documentales. México. Limusa.

Conti, A., Oltramari, R., \& Navarro, L. (2007). Examination of temporomandibular disorders in the orthodontic patient: a clinical guide. Journal of Applied Oral Science, 15(1), 77-82. Recuperado el 2021 de Oct de 2021

Davila, A. (2015). Diccionario de Términos Científicos. . Caracas: Editorial Oasis.

Decker, A., \& Kohault, C. (2008). Traitements des dysfonctions temporomandibulaires,", P. Canal and. In Orthodontie de l'adulte Masson, 231-238. Recuperado el 22 de Oct de 2021

Gutiérrez, L. (18 de Mar de 2020). Tu Salud Guia. Recuperado el 22 de Oct de 2021, de https://tusaludguia.com/rc-relacion-centrica/

Hamata, M., Zuim, P., \& Garcia, A. (2009). Comparative evaluation of the efficacy of occlusal splints fabricated in centric relation or maximum intercuspation in temporomandibular disorders patients. Journal of Applied Oral Science, 17(1), 32-38.

Kirveskari, P., \& Alanen, D. (2009). Paradigms and EBD. Journal of Orofacial Pain , 23(4), 299-300. Recuperado el 21 de Oct de 2021 
López, V., Gómez, G., \& Canseco, J. (Sep de 2004). Evaluación clínica de la disfunción temporomandibular antes del tratamiento ortodóncico. Revista Odontológica Mexicana, 8 (3), 80-89. Recuperado el 22 de Oct de 2021, de https://www.medigraphic.com/pdfs/odon/uo-2004/uo043f.pdf

Michelotti, A., \& Lodice, G. (2010). The role of orthodontics in temporomandibular disorders. Journal of Oral Rehabilitation, 37(6), 411-429. doi:doi: 10.1111/j.1365-2842.2010.02087.x

Okeson, J., \& Leeuw, R. (2011). Differential diagnosis of temporomandibular disorders and other orofacial pain disorders. Dental Clinics of North America, 55(1), 105-120. doi:DOI:10.5772/31316

Orozco, A., \& Arroyo, G. (Nov de 2008). Relación céntrica: revisión de conceptos y técnicas para su registro. Parte I. Avances Odontoestomatol, 24(6). Recuperado el 22 de Oct de 2021, de https:// scielo.isciii.es/scielo.php?script=sci_arttext\&pi$d=$ S0213-12852008000600003

Orthlieb, J., Brocard, D., \& Schittly, B. (2006). Occlusodontie Pratique. Collection JPIO, CDP. Recuperado el 22 de Oct de 2021, de https://www. unitheque.com/occlusodontie-pratique/jpio/cdp/ Livre/14410
Rinchuse, D., \& Kandasamy, S. (2006). Centric relation: a historical and contemporary orthodontic perspective,. Journal of the American Dental Association, 137(4), 494-500.

SEDCYDO. (20 de Nov de 2015). Sociedad Española de Disfunción Craneomandibular y Dolor Orofacial. Recuperado el 22 de Oct de 2021, de www. sedcydo.com

Turp, J., \& Schindler, H. (2012). The dental occlusion as a suspected cause for TMDs: epidemiological and etiological considerations. Journal of Oral Rehabilitation, vol. 39, pp. 502-, 39, 502-512. Recuperado el 21 de Oct de 2021

Venegas, C., Farfán, C., \& Fuentes, R. (2021). Posiciones Mandibulares de Referencia Clínica. Una Descripción Narrativa. Int. J. Odontostomat, 15(2), 387-396. Obtenido de https://www.scielo.cl/pdf/ ijodontos/v15n2/0718-381X-ijodontos-15-02-387. pdf

Winocur, E., \& Gavish, T. (2001). "Oral habits among adolescent girls and their association with symptoms of temporomandibular disorders. Journal of Oral Rehabilitation, 287, 624-629. Recuperado el 21 de Oct de 2021

\section{CITAR ESTE ARTICULO:}

Rosero Mendoza, J. I., Mendoza Rodríguez, F. A., Núñez García, C. L., \& Rosero Mendoza, J. C. (2021). Disfunción craneomandibular y su relación con la ortodoncia u oclusión. RECIMUNDO, 5(4), 181-190. https://doi.org/10.26820/ recimundo/5.(4).oct.2021.181-190 